\title{
Safety of a high-dose house dust mite allergoid in pediatric patients
}

\section{Clinical data from real-life situation in a medical practice}

\section{Dieter Ullrich}

Received: 22 April 2018 / Accepted: 3 October 2018 / Published online: 6 November 2018 (C) The Author(s) 2018

Keywords House-dust mite allergoid · Safety · Children · Adolescents · Daily practice

\section{To the editors}

\section{Background}

I would like to add the experience from an Ear-NoseThroat (ENT) medical practice in the north of Germany to the publication in the Allergo Journal International that addressed the safety of subcutaneous immunotherapy (SCIT) with a high-dose house dust mite (HDM) allergoid using data from pooled clinical trials [1].

Safety and tolerability of allergen immunotherapy (AIT) with this SCIT product in children and adolescents with HDM allergic rhinoconjunctivitis with or without asthma are reported.

\section{Methods}

According to prospectively defined clinical criteria, the records of all children and adolescents between 5 and $\leq 17$ years (median age 12 years; female 26, male 38 ) treated with the HDM-AIT in the ENT practice between 2005 and $2017(n=64)$ were retrospectively analysed. No file was excluded.

\section{Author contribution D. Ullrich developed the design, performed the data analysis, supervised the writing of the manuscript and reviewed, commented, and approved the final manuscript.}

\section{Dr. D. Ullrich $(\bowtie)$}

Medical practice for ear, nose and throat medicine, Wedemarkstraße 83, 30900 Wedemark, Germany Ullrich-Dieter@t-online.de

\section{Results}

Treated patients had a history of allergic symptoms (nasal obstruction especially during the night, recurrent bronchitis or asthma symptoms, sleep disturbance and snoring), a positive Skin Prick Test (SPT) to HDM ( $>3 \mathrm{~mm})$ and an IgE $\geq$ class 3 to Dermatophagoides pteronyssinus (D.pt.) and Dermatophagoides farinae (D.f). In 40 cases a nasal provocation test with HDM was performed.

16 of the 64 paediatric patients suffered from additional asthma (GINA II, III) [2]; 28 patients were polysensitized (Table 1) and 21 received concomitant SCIT (grass/rye, early-blossoming trees, cat) on the same day. The waiting period between injections was $15 \mathrm{~min}$.

Overall, the paediatric patients received 1942 injections with the HDM allergoid, median 34 injections/patient. In 17 injections $(0.88 \%, 13$ patients), a systemic adverse drug reaction (ADR) was observed ( $12 \times$ cough $/$ mild dyspnoea, $2 \times$ nasal obstruction or flu-like symptoms, $3 \times$ swelling of the arm/urticaria; Table 2). The 17 reactions were of grade 1 and 2 according to clinical judgement. 8 patients with ADR got two simultaneous AITs (in one patient SCIT for cat and HDM). None of these AITs were stopped because of the ADR; therapy was continued with an unchanged dose without pre-medication or changes in intervals.

There were no significant differences with respect to ADRs in the different age groups. The relative number of systemic ADRs per injection was 0.97 in children (5-12 years) compared to 0.74 in adolescents (13- $\leq 17$ years). 9 of the 17 ADRs were late phase reactions after more than $60 \mathrm{~min}$ (up to $6 \mathrm{~h}$ ) with mild dyspnoea, urticaria and swelling of the arm. In none of the late events was medical support requested or intervention necessary. 
Table 1 Baseline characteristics

\begin{tabular}{|c|c|c|c|c|c|c|c|c|}
\hline \multirow[t]{2}{*}{ Age (years) } & \multicolumn{2}{|l|}{$5-6$} & \multicolumn{2}{|l|}{$7-12$} & \multicolumn{2}{|c|}{$13-17$} & \multicolumn{2}{|l|}{ Total } \\
\hline & Male & Female & Male & Female & Male & Female & Male & Female \\
\hline Number of patients $(n)$ & 3 & 1 & 20 & 12 & 15 & 13 & 38 & 26 \\
\hline Allergic rhinitis (AR) & 3 & 1 & 20 & 12 & 15 & 13 & 38 & 26 \\
\hline Allergic asthma & - & 1 & 4 & 6 & 3 & 2 & 7 & 9 \\
\hline \multicolumn{9}{|l|}{ Asthma medication } \\
\hline Controller & 0 & 0 & 3 & 4 & 2 & 1 & 5 & 5 \\
\hline Reliever & - & 1 & 1 & 2 & 1 & 1 & 2 & 4 \\
\hline Positive nasal provocation HDM & 3 & - & 10 & 4 & 9 & 8 & 22 & 12 \\
\hline Results not conclusive & - & 1 & 1 & 2 & 1 & 1 & 2 & 4 \\
\hline 1 additional allergy & 1 & 1 & 5 & 2 & 2 & 6 & & 17 \\
\hline 2 additional allergies & - & - & 2 & 1 & 4 & 2 & & 9 \\
\hline 3 additional allergies & - & - & 1 & - & 1 & - & & 2 \\
\hline Grass/Rye allergy & & 2 & & 12 & & 10 & & 24 \\
\hline Tree (early-blossoming) allergy & & - & & 5 & & 7 & & 12 \\
\hline Cat allergy & & - & & 4 & & 4 & & 8 \\
\hline Additional SCIT & - & - & 7 & 3 & 3 & 8 & & 21 \\
\hline
\end{tabular}

Table 2 Systemic ADRs per age group

\begin{tabular}{|c|c|c|c|c|c|c|c|c|}
\hline \multirow[t]{2}{*}{ Age (years) } & \multicolumn{2}{|l|}{$5-6$} & \multicolumn{2}{|l|}{$7-12$} & \multicolumn{2}{|c|}{$13-17$} & \multicolumn{2}{|l|}{ Total } \\
\hline & Male & Female & Male & Female & Male & Female & Male & Female \\
\hline Number of patients $(n)$ & 3 & 1 & 20 & 12 & 15 & 13 & 38 & 26 \\
\hline Number of injections $(n)$ & 81 & 28 & 646 & 377 & 466 & 344 & 1193 & 749 \\
\hline Number of systemic ADRs $(n)$ & 1 & 0 & 5 & 5 & 2 & 4 & 8 & 9 \\
\hline Systemic ADRs per injection (\%) & 1.23 & 0 & 0.77 & 1.33 & 0.43 & 1.16 & 0.67 & 1.20 \\
\hline
\end{tabular}

43 paediatric patients finalized their regular 3-year AIT, in 7 patients the analysis was performed before the end of treatment and in 14 patients AIT was performed in another medical practice or stopped prematurely. 80 to $90 \%$ of participants evaluated the treatment as very effective.

\section{Discussion}

Data of SCIT AIT in mite-allergic children and adolescents are rare and populations in clinical trials are often very well selected so that data from daily practice, real-life situations and unselected patients are important.

In this real-life observation, data of all paediatric patients treated with the high-dose HDM allergoid in an ENT practice (2005 to 2017) are reported. The number of polysensitized patients ( $44 \%$ of all patients; $86 \%$ of these were allergic to HDM and grass/rye, $43 \%$ to HDM and early-blossoming trees, $29 \%$ to HDM and cat) and patients treated with two SCIT AITs simultaneously (33\%) were comparable to recent literature [3]. We observed systemic ADRs in 13 of the 64 paediatric patients $(20 \%)$. In the publication of Klimek et al. [1], systemic reactions were seen in $12.8 \%$ of children and $7.7 \%$ of adolescents. Reasons for this difference could be the simultaneous AIT with other allergens in $33 \%$ of patients, the high number of injections per patient, and the fact that of 16 asthmatic patients, only $63 \%$ were treated with controller medication. 9 of 17 ADR's appeared within a period of $60 \mathrm{~min}$ to $6 \mathrm{~h}$ after injection. Such a long follow-up period is unusual. And we also observed a lower number of ADRs in adolescents compared to children.

According to our data, the tolerability of AIT with a high-dose HDM allergoid is a safe treatment strategy in pediatric patients under real-life conditions.

Acknowledgements The author acknowledges medical writing and editorial support for the preparation of this LTE from Dr. A. Narkus, MC-Narkus GmbH, Germany.

Funding Financial support exclusively for medical writing of this article was provided by Allergopharma GmbH \& Co. KG.

Conflict of interest D. Ullrich declares that he has no competing interests.

Open Access This article is distributed under the terms of the Creative Commons Attribution 4.0 International License (http://creativecommons.org/licenses/by/4.0/), which permits unrestricted use, distribution, and reproduction in any medium, provided you give appropriate credit to the original author(s) and the source, provide a link to the Creative Commons license, and indicate if changes were made. 


\section{References}

1. Klimek L, Fox G-C, Thum-Oltmer S. SCIT with a high-dose house dust mite allergoid is well tolerated: safety data from pooled clinical trials and more than 10-years of daily practice analyzed in different subgroups. Allergo J Int. 2018;27:131-9.

2. GINA Report, Global Strategy for Asthma Management and Prevention. https://ginasthma.org/2018-gina- report-global-strategy-for-asthma-management-andprevention. Accessed Oct 2018

3. Wahn U, Calderon MA, Demoly P. Real-life clinical practice and management of polysensitized patients with respiratory allergies: A large, global survey of clinicians prescribing allergen immunotherapy. Expert Rev Clin Immunol. 2017;13:283-9. 